\title{
Nondissipative Second-Order Transport, Spin, and Pseudogauge Transformations in Hydrodynamics
}

\author{
Shiyong Li®, ${ }^{*}$ Mikhail A. Stephanov, ${ }^{\dagger}$ and Ho-Ung Yee ${ }^{\ddagger}$ \\ Physics Department, University of Illinois at Chicago, Chicago, Illinois 60607, USA
}

(Received 18 December 2020; revised 1 June 2021; accepted 13 July 2021; published 20 August 2021)

\begin{abstract}
We derive a set of nontrivial relations between second-order transport coefficients which follow from the second law of thermodynamics upon considering a regime close to uniform rotation of the fluid. We demonstrate that an extension of hydrodynamics by spin variable is equivalent to modifying conventional hydrodynamics by a set of second-order terms satisfying the relations we derived. We point out that a novel contribution to the heat current orthogonal to vorticity and temperature gradient reminiscent of the thermal Hall effect is constrained by the second law.
\end{abstract}

DOI: 10.1103/PhysRevLett.127.082302

Introduction.-Relativistic hydrodynamics [1] is an effective description, at large distance and timescales, of systems in local thermodynamic equilibrium parametrized by slowly varying profiles of four-velocity $u^{\mu}(x)\left(u_{\mu} u^{\mu}=-1\right)$, local temperature $T(x)$, and chemical potential $\mu(x)$ for a conserved charge. The system of equations based on the conservation laws is closed, and all dynamic information about the system in the hydrodynamic regime is contained in the hydrodynamic variables. Relativistic hydrodynamics has been successful in many branches of physics, in particular, in describing the dynamical evolution of the fireball created in relativistic heavy-ion collisions (RHIC) [2,3].

Recent developments include interesting attempts to incorporate spin polarization of microscopic constituents as an additional hydrodynamic variable characterizing the system, which led to consideration of "spin hydrodynamics" [4-6]. This is motivated by the importance of spin observables in many applications of hydrodynamics in condensed matter as well as nuclear physics. Specifically, each event in noncentral RHIC collisions carries a significant amount of initial orbital angular momentum, some of which is transferred to the spin polarization of observed hadrons [7-13]. However, in the strict sense of hydrodynamics, spin polarization of plasma constituents should also be in local equilibrium, and must be determined by conventional hydrodynamic variables [14].

In this Letter we assume the standard local equilibrium, and show that the spin hydrodynamics and the conventional hydrodynamics are two equivalent descriptions of the same

Published by the American Physical Society under the terms of the Creative Commons Attribution 4.0 International license. Further distribution of this work must maintain attribution to the author(s) and the published article's title, journal citation, and DOI. Funded by SCOAP . system. This not only reconciles the two formulations, but also leads us to find new constraints for certain transport coefficients in conventional second-order hydrodynamics.

The central question we answer in this Letter is the meaning of pseudogauge transformations [16-19] in spin hydrodynamics. Since hydrodynamics is based on local thermodynamics, this question can only be answered after properly addressing how thermodynamics transform under pseudogauge transformations. We show the equivalence of local thermodynamics between the spin and conventional hydrodynamics, which requires us to generalize pseudogauge transformation to currents of entropy and conserved charge. We use these results to prove the equivalence between spin hydrodynamics and conventional hydrodynamics. In particular, we find that the ideal limit of spin hydrodynamics is equivalent to conventional hydrodynamics with certain nondissipative second-order transport coefficients. Moreover, five of these second-order transport coefficients are uniquely determined by two thermodynamic functions, one of which appears as the spin susceptibility in the spin hydrodynamics description.

The existence of such constraints on certain secondorder transport coefficients is an interesting fact by itself, independent of its physics connection to the spin hydrodynamics. Within conventional hydrodynamics, we show that the same constraints can be derived directly using the second law of thermodynamics, and are therefore universal. Our derivation is based on a new power counting scheme for gradients of hydrodynamic variables, motivated by considering small deviations from one of the equilibrium states of uniformly rotating fluid, which exist due to the conservation of total angular momentum.

We consider dissipative gradients of fluid velocity and of $\alpha=\mu / T$, as being much smaller than the vorticity and the temperature gradients neither of which appear in the entropy production rate at leading order in gradients. 
This allows us to reorganize the naive gradient expansion in the entropy production rate and to derive a set of nontrivial constraints on certain second-order transport coefficients by applying the second law of thermodynamics. Our method should be more generally applicable to some higher-order transport coefficients, as well as to transport coefficients involving external electromagnetic fields, but we leave such generalizations to future work.

Although similar constraints have been found for chargeless fluid [20-22] and charged fluid in Ref. [23] using a different approach, the constraints in Ref. [23] appear to be less stringent, leaving four unconstrained parameters in constrast to the two coefficients we find. It would be interesting to establish a relationship between the constraints we derive and the ones in Ref. [23], which appears to be a nontrival task due to the difference in choices of variables and frames (we use the conventional Landau frame).

Nondissipative second-order hydrodynamics. - Guided by the observation that vorticity in a uniformly rotating fluid can take arbitrary values without entropy production, we consider fluid states where vorticity and temperature gradients $\omega_{\mu \nu}=\frac{1}{2}\left(\partial_{\mu}^{\perp} u_{\nu}-\partial_{\nu}^{\perp} u_{\mu}\right), \partial_{\mu}^{\perp} \beta$, while still being small, are larger than other, dissipative gradients $\theta_{\mu \nu}=$ $\frac{1}{2}\left(\partial_{\mu}^{\perp} u_{\nu}+\partial_{\nu}^{\perp} u_{\mu}\right)$ and $\partial_{\mu}^{\perp} \alpha$, where $\partial_{\mu}^{\perp} \equiv \Delta_{\mu \nu} \partial^{\nu} \quad$ with $\Delta_{\mu \nu}=u_{\mu} u_{\nu}+g_{\mu \nu}, \beta \equiv 1 / T$, and $\alpha \equiv \beta \mu$. To this end, we introduce the following power counting scheme: $\omega_{\mu \nu} \sim \epsilon_{\omega}$, $\partial_{\mu}^{\perp} \beta \sim \epsilon^{\prime}$, and $\theta_{\mu \nu} \sim \partial_{\mu}^{\perp} \alpha \sim \epsilon$, while any further spatial derivative on $\left(\omega_{\mu \nu}, \beta\right)$ and $\left(\theta_{\mu \nu}, \alpha\right)$ brings an extra $\epsilon^{\prime}$ and $\epsilon$, respectively. For example, $\partial_{\rho}^{\perp} \omega_{\mu \nu} \sim \epsilon_{\omega} \epsilon^{\prime}, \partial_{\mu}^{\perp} \partial_{\nu}^{\perp} \beta \sim \epsilon^{\prime 2}$, and $\partial_{\rho}^{\perp} \theta_{\mu \nu} \sim \partial_{\mu}^{\perp} \partial_{\nu}^{\perp} \alpha \sim \epsilon^{2}$. In addition, we consider spatial gradients of thermal vorticity to be of the same order as the dissipative gradients, i.e., $\partial_{\nu}^{\perp}\left(\beta \omega^{\mu}\right) \sim \epsilon_{\omega} \epsilon$ rather than $\epsilon_{\omega} \epsilon^{\prime}$, which means $\partial_{\nu}^{\perp} \omega^{\mu}=-\left(\partial_{\nu}^{\perp} \beta\right) \omega^{\mu} / \beta+\mathcal{O}\left(\epsilon_{\omega} \epsilon\right)$. From this and the ideal equation of motion, one can show that $\partial_{\mu} \omega^{\mu} \sim \omega^{\mu} \partial_{\mu} \beta \sim \epsilon_{\omega} \epsilon$.

We then invoke the hierarchy $\epsilon^{\prime 2} \ll \epsilon \ll \epsilon_{\omega} \epsilon^{\prime} \ll$ $\epsilon_{\omega}^{2} \ll \epsilon^{\prime} \ll \epsilon_{\omega} \ll 1$. As we will see, this allows us to focus on the vorticity related terms arising from certain secondorder transport coefficients as the leading contributions to the entropy production rate up to order $\epsilon_{\omega} \epsilon^{\prime} \epsilon$, while the dissipative terms from first-order transport coefficients are of the order $\epsilon^{2} \ll \epsilon_{\omega} \epsilon^{\prime} \epsilon$, and are thus subleading. Note that $\epsilon_{\omega} \epsilon^{\prime} \epsilon$ would naively be of higher order than $\epsilon^{2}$ in the conventional gradient expansion. By careful inspection of all possible terms in the entropy production rate, potentially larger terms of $\epsilon_{\omega}^{4}, \epsilon_{\omega}^{3} \epsilon^{\prime}$, and $\epsilon_{\omega}^{2} \epsilon^{\prime 2}$ can be shown to be absent in parity even plasma that we focus on in this Letter. Then the second law of thermodynamics, i.e., the nonnegativity of entropy production, should be applied to these leading contributions involving second-order transport coefficients.

We write the general parity even constitutive relations for symmetric energy-momentum tensor, as well as for charge and entropy currents:

$$
\begin{gathered}
T^{\mu \nu}=(\varepsilon+p) u^{\mu} u^{\nu}+p g^{\mu \nu}+\Delta T^{\mu \nu}, \\
j^{\mu}=n u^{\mu}+\Delta j^{\mu}, \\
s^{\mu}=s u^{\mu}+\Delta s^{\mu},
\end{gathered}
$$

where $\Delta T^{\mu \nu}, \Delta j^{\mu}$, and $\Delta s^{\mu}$ contain all relevant second-order terms in our hierarchy,

$$
\begin{gathered}
\Delta T^{\mu \nu}=a_{0} \Delta^{\mu \nu} \omega^{\lambda \rho} \omega_{\lambda \rho}+a_{1} \omega_{\lambda}^{\mu} \omega^{\lambda \nu}, \\
\Delta j^{\mu}=c_{1} \Delta_{\rho}^{\mu} \partial_{\nu} \omega^{\nu \rho}+c_{2} \omega^{\mu \nu} \partial_{\nu} \beta, \\
\Delta s^{\mu}+\alpha \Delta j^{\mu}=b_{1} \Delta_{\rho}^{\mu} \partial_{\nu} \omega^{\nu \rho}+b_{2} \omega^{\mu \nu} \partial_{\nu} \beta+b_{3} \omega^{\mu \nu} \partial_{\nu} \alpha,
\end{gathered}
$$

with seven second-order transport coefficients $\left\{a_{i}, b_{i}, c_{i}\right\}$. We do not need to include the first-order transport terms as explained above, and we omit other possible second-order terms, such as $\partial_{\mu}^{\perp} \beta \partial_{\nu}^{\perp} \beta$ in $\Delta T^{\mu \nu}$ and $\omega^{\mu \nu} \partial_{\nu} \alpha$ in $\Delta j^{\mu}$, that do not contribute to the entropy production rate to order $\epsilon_{\omega} \epsilon^{\prime} \epsilon$, and whose coefficients are thus not constrained by our method. We also remark that one could put a purely spatial gradient $\Delta_{\rho}^{\mu} \Delta_{\nu}^{\gamma} \partial_{\gamma} \omega^{\nu \rho}$ in place of $\Delta_{\rho}^{\mu} \partial_{\nu} \omega^{\nu \rho}$, but this would be equivalent up to a redefinition of $\left\{b_{2}, c_{2}\right\}$ due to the ideal equations of motion and the thermodynamic relation $\beta d p=-w d \beta+n d \alpha$.

Introducing $\omega^{\mu} \equiv \frac{1}{2} \epsilon^{\mu \nu \alpha \beta} u_{\nu} \omega_{\alpha \beta}$ and using the identity

$\omega_{\mu} D \omega^{\mu}=\omega^{\mu \nu} \frac{\left(\partial_{\mu} \varepsilon\right)\left(\partial_{\nu} p\right)}{2 w^{2}}-\omega_{\mu} \omega^{\mu} \frac{D p}{w}-\omega_{\alpha}^{\mu} \omega^{\alpha \nu} \theta_{\mu \nu}$

which follows from the ideal equations of motion, where $w=\varepsilon+p$ and $D \equiv u^{\mu} \partial_{\mu}$, one finds the entropy production rate up to $O\left(\epsilon_{\omega} \epsilon^{\prime} \epsilon\right)$ given by

$$
\begin{aligned}
\partial_{\mu} s^{\mu}= & C^{(1)} \omega_{\nu} \omega^{\nu} \theta+C^{(2)}\left(\partial_{\nu}^{\perp} \omega^{\nu \mu}\right) \partial_{\mu}^{\perp} \alpha+C^{(3)}\left(\partial_{\nu}^{\perp} \omega^{\nu \mu}\right) \partial_{\mu}^{\perp} \beta \\
& +C^{(4)}\left(\partial_{\mu} \beta\right) \omega^{\mu \nu}\left(\partial_{\nu} \alpha\right)+C^{(5)} \theta_{\mu \nu} \omega_{\alpha}^{\mu} \omega^{\alpha \nu},
\end{aligned}
$$

where $\theta \equiv \theta_{\mu}^{\mu}=\partial_{\mu} u^{\mu}$ and $C^{(i)}$ are given by

$$
\begin{aligned}
C^{(1)}= & -2\left(a_{0} \beta+b_{1}+2 b_{1} c_{s}^{2}+b_{2} w \beta_{\varepsilon}+b_{3} \alpha_{p} w c_{s}^{2}\right), \\
C^{(2)}= & \left(\frac{\partial b_{1}}{\partial \alpha}\right)_{\beta}+b_{3}-c_{1}, \quad C^{(3)}=\left(\frac{\partial b_{1}}{\partial \beta}\right)_{\alpha}+b_{2}, \\
C^{(4)}= & \frac{b_{3}}{\beta}+\left(\frac{\partial b_{3}}{\partial \beta}\right)_{\alpha}+\frac{n}{\beta w}\left(\frac{\partial b_{1}}{\partial \beta}\right)_{\alpha}+\frac{1}{\beta}\left(\frac{\partial b_{1}}{\partial \alpha}\right)_{\beta} \\
& +2 b_{1} \frac{\partial}{\partial \beta}\left(\frac{n}{\beta w}\right)_{\alpha}+\frac{b_{2} n}{\beta w}-\left(\frac{\partial b_{2}}{\partial \alpha}\right)_{\beta}-\frac{c_{1}}{\beta}+c_{2}, \\
C^{(5)}= & a_{1} \beta+4 b_{1},
\end{aligned}
$$

where $c_{s}^{2}=(\partial p / \partial \varepsilon)_{s / n}, \quad \alpha_{p}=(\partial \alpha / \partial p)_{s / n}, \quad$ and $\beta_{\varepsilon}=$ $(\partial \beta / \partial \varepsilon)_{s / n}$ are thermodynamic derivatives taken with $s / n$ fixed, which appear naturally due to the ideal equations of motion, $D(s / n)=0$.

All five terms in Eq. (8) are independent and can have either sign for generic initial conditions. The second law of 
thermodynamics thus requires that all $C^{(i)}$ vanish. This gives five constraints for seven unknowns $\left\{a_{i}, b_{i}, c_{i}\right\}$, which determines them up to two free functions. Choosing $a_{0}$ and $a_{1}$ as two given functions, one can solve for the other five transport coefficients without any integration, proceeding in the following order:

$$
\begin{aligned}
& b_{1}=-\frac{\beta a_{1}}{4}, \quad b_{2}=-\left(\frac{\partial b_{1}}{\partial \beta}\right)_{\alpha}, \\
& b_{3}=\frac{1}{\alpha_{p} w c_{s}^{2}}\left[w \beta_{\epsilon}\left(\frac{\partial b_{1}}{\partial \beta}\right)_{\alpha}-b_{1}-2 b_{1} c_{s}^{2}-\beta a_{0}\right], \\
& c_{1}=b_{3}+\left(\frac{\partial b_{1}}{\partial \alpha}\right)_{\beta}, \\
& c_{2}=-\left(\frac{\partial c_{1}}{\partial \beta}\right)_{\alpha}-2 b_{1} \frac{\partial}{\partial \beta}\left(\frac{n}{\beta w}\right)_{\alpha} .
\end{aligned}
$$

As a nontrivial check of these relations we can consider conformal theory, such as the strongly coupled conformal plasma described by the AdS-CFT correspondence for which some of the coefficients have been calculated in Ref. [24]. Conformal invariance imposes certain constraints on some of the thermodynamic quantities, such as $w=4 \varepsilon / 3, c_{s}^{2}=1 / 3, \beta_{\varepsilon}=-\beta /(4 \varepsilon)$, and $\alpha_{p}=0$, as well on transport coefficients: $a_{1}=3 a_{0}$ and $\left(\partial b_{1} / \partial \beta\right)_{\alpha}=$ $-b_{1} / \beta$. Substituting into Eq. (10b) we find that it is satisfied for any $b_{3}$ because, while $\alpha_{p}=0$, also the expression in the square brackets nontrivially vanishes, provided $b_{1}$ is given by Eq. (10a). Furthermore, conformal invariance requires $[\partial(n / \beta w) / \partial \beta]_{\alpha}=0$. Substituting into Eq. (10c), we find a relationship between $c_{1}$ and $c_{2}$ which coincides with a nontrivial constraint imposed by conformal Weyl symmetry [24,25]. Finally, solving Eqs. (10a) and (10c) we can now predict the values of $b_{1}, b_{2}$, and $b_{3}$ which have not been calculated in Ref. [24], in terms of $a_{1}$ and $c_{1}$ which have been calculated.

Spin hydrodynamics. - Spin hydrodynamics is based on the energy-momentum tensor $\Theta^{\mu \nu}$ and the rank-3 tensor $S^{\mu \alpha \beta}=-S^{\mu \beta \alpha}$ of spin current. The total angular momentum tensor consists of the orbital and the spin parts, $J^{\mu \alpha \beta}=$ $\left(x^{\alpha} \Theta^{\mu \beta}-x^{\beta} \Theta^{\mu \alpha}\right)+S^{\mu \alpha \beta}$, and the formalism needs the additional conservation law, $\partial_{\mu} J^{\mu \alpha \beta}=0$, corresponding to the introduction of additional spin degrees of freedom. This relates the antisymmetric part of $\Theta^{\mu \nu}$ to nonconservation of spin due to the spin-orbit exchange of angular momentum: $\Theta^{\mu \nu}-\Theta^{\nu \mu}=-\partial_{\alpha} S^{\alpha \mu \nu}$.

The constitutive relations are given by

$$
\begin{aligned}
\Theta^{\mu \nu}= & \varepsilon u^{\mu} u^{\nu}+p \Delta^{\mu \nu}+\left(u^{\mu} q^{\nu}+u^{\nu} q^{\mu}\right)+\tau^{\mu \nu} \\
& -\frac{1}{2} \partial_{\alpha} S^{\alpha \mu \nu}, \\
& j^{\mu}=n u^{\mu}+\tau^{\mu}, \quad S^{\mu \alpha \beta}=u^{\mu} S^{\alpha \beta}+\sigma^{\mu \alpha \beta},
\end{aligned}
$$

where we do not assume that $u^{\mu}$ is the Landau frame, $q^{\mu}$ $\left(u^{\mu} q_{\mu}=0\right)$ is a contribution to energy current, $S^{\mu \nu}$ is the spin density in the local rest frame satisfying the Frenkel condition $u_{\mu} S^{\mu \nu}=0$, and $\left(\tau^{\mu \nu}, \tau^{\mu}, \sigma^{\mu \alpha \beta}\right)$ are dissipative gradient corrections. We will not be concerned with these dissipative terms in our subsequent discussion of an ideal limit, because their inclusion will not affect our main conclusion.

Writing the entropy current as $s^{\mu}=s u^{\mu}+\Delta s^{\mu}$, $\left(u_{\mu} \Delta s^{\mu}=0\right)$ and adding $0=\beta_{\nu} \partial_{\mu} \Theta^{\mu \nu}+\alpha \partial_{\mu} j^{\mu}$ to $\partial_{\mu} s^{\mu}$ we obtain the following expression for the entropy production rate:

$$
\begin{aligned}
\partial_{\mu} s^{\mu}= & {\left[D s-\beta D \varepsilon+\alpha D n+\frac{1}{2} \beta \omega_{\mu \nu} D S^{\mu \nu}\right] } \\
& +\theta\left[s-\beta(\varepsilon+p)+\alpha n+\frac{1}{2} \beta \omega_{\mu \nu} S^{\mu \nu}\right]-\beta \tau^{\mu \nu} \theta_{\mu \nu}-\tau^{\mu} \partial_{\mu} \alpha \\
& +\partial_{\mu}\left[\Delta s^{\mu}-\frac{1}{2} \beta_{\nu} \partial_{\alpha} S^{\alpha \mu \nu}-\beta q^{\mu}+\alpha \tau^{\mu}+\frac{1}{2}\left(\partial_{\rho} \beta_{\delta}\right) \sigma^{\mu \rho \delta}\right] \\
& +\left[\left(-\beta D u_{\nu}+\partial_{\nu} \beta\right)\left(q^{\nu}-\frac{1}{2 \beta} S^{\nu \rho} \partial_{\rho} \beta\right)\right] \\
& -\frac{1}{2}\left(\partial_{\alpha} \partial_{\mu} \beta_{\nu}\right) \sigma^{\alpha \mu \nu}
\end{aligned}
$$

where $\beta_{\nu} \equiv \beta u_{\nu}$.

There exists an ideal limit of spin hydrodynamics where the right-hand side of Eq. (13) vanishes. The vanishing of the first two square brackets leads to the following thermodynamics relation [26]:

$$
\begin{aligned}
d s & =\beta d \varepsilon-\alpha d n-\frac{\beta}{2} \gamma_{\mu \nu} d S^{\mu \nu}, \\
s & =\beta(\varepsilon+p)-\alpha n-\frac{\beta}{2} \gamma_{\mu \nu} S^{\mu \nu},
\end{aligned}
$$

where the entropy density is a function of $\varepsilon, n$, and $S^{\mu \nu}$, with the spin potential being equal to the fluid vorticity in local equilibrium, $\gamma_{\mu \nu}=\omega_{\mu \nu}$. We emphasize that the spin density should be fixed by the spin potential as a thermodynamic relation in equilibrium, i.e., $S^{\mu \nu}=\chi \gamma^{\mu \nu}$ with the spin susceptibility $\chi$ [27]. This determines the spin density in terms of hydrodynamic variables, $S^{\mu \nu}=\chi \omega^{\mu \nu}$.

Vanishing of other terms requires

$\Delta S^{\mu}=\frac{1}{2} \beta_{\nu} \partial_{\alpha}\left(u^{\alpha} S^{\mu \nu}\right)+\beta q^{\mu}-\alpha \tau^{\mu}-\frac{1}{2}\left(\partial_{\rho} \beta_{\delta}\right) \sigma^{\mu \rho \delta}$

and the following relation:

$$
q^{\mu}-\frac{w}{n} \tau^{\mu}=\frac{1}{2 \beta} S^{\mu \nu} \partial_{\nu} \beta=\frac{\chi}{2 \beta} \omega^{\mu \nu} \partial_{\nu} \beta .
$$

Equation (16) is independent of the choice of the hydrodynamic frame $u^{\mu}$. However, one can show, by introducing an impurity [28], that $\tau^{\mu}$ vanishes in the 
"no-drag frame" [29]. This is a nontrivial example, similar to the Chiral Vortical Effect $[28,29]$, where the entropy flows past a static impurity without generating a drag. One could refer to this nondissipative heat current we find as the vorticity driven thermal Hall effect.

As a nontrivial check of Eq. (16) we can calculate the heat current in the no-drag frame for the microscopic chiral kinetic theory of massless Dirac fermion. As detailed in Ref. [30], we choose the fluid rest frame as the spin frame $n_{\mu}=u_{\mu}$ so that the Frenkel condition is satisfied. With $n^{\mu}=(1,0,0,0)$, the spin density $s$ is proportional to the axial current, $s^{i}=\hbar j_{5}^{i}=\hbar \bar{\psi} \gamma^{i} \gamma_{5} \psi$. Therefore, $s=\int_{p, \lambda} \hbar \lambda j_{p}$, where $\boldsymbol{j}_{p}$ is the phase space (Liouville) current and $\int_{\boldsymbol{p}, \lambda} \equiv \sum_{\lambda= \pm 1 / 2} \int d^{3} \boldsymbol{p} /(2 \pi \hbar)^{3}$ includes the sum over helicities $\lambda$. According to Ref. [30], to order $\mathcal{O}(\hbar)$, $\boldsymbol{j}_{p}=\left[\hat{\boldsymbol{p}}-\left(\hbar \lambda / p_{0}\right) \hat{\boldsymbol{p}} \times \boldsymbol{\nabla}\right] f_{\mathrm{eq}}$, where $p_{0}=|\boldsymbol{p}|$. The second term in $\boldsymbol{j}_{p}$ not only accounts for $2 / 3$ of the Chiral Vortical Effect [31], but also plays an important role below to give the correct spin density. For uniformly rotating (shear-free) fluid in thermodynamic equilibrium, the particle distribution function in the no-drag frame takes the form [29,30] $f_{\text {eq }}=1 /\left(\exp \left\{\beta\left[-p_{\mu} u^{\mu}+(1 / 2) S_{n}^{\mu \nu} \omega_{\mu \nu}\right]\right\}+1\right)$, where $S_{n}^{\mu \nu}=$ $\lambda \epsilon^{\mu \nu \alpha \beta} p_{\alpha} n_{\beta} /\left(p_{\mu} n^{\mu}\right)$ and $\mu=0$ for simplicity. The spin density $S^{i j}$ can then be computed as

$$
S^{i j}=\epsilon^{i j k} s_{k}=\frac{\omega^{i j}}{24 \hbar \beta^{2}}+O\left(\hbar^{0}\right) .
$$

On the other hand, the energy-momentum tensor is given by $\Theta^{\mu \nu}=\int_{p, \lambda} j_{p}^{\mu} p^{\nu}$. Using the known result for $j_{p}^{\mu}$, now up to $O\left(\hbar^{2}\right)$ from Ref. [32],

$$
\begin{aligned}
\boldsymbol{j}_{p}= & {\left[\hat{\boldsymbol{p}}-\frac{\hbar \lambda}{p_{0}} \hat{\boldsymbol{p}} \times \boldsymbol{\nabla}+\frac{(\hbar \lambda)^{2}}{p_{0}^{2}}(\hat{\boldsymbol{p}} \times \boldsymbol{\nabla}) \times \boldsymbol{\nabla}\right] f_{\mathrm{eq}} } \\
& +(\hbar \lambda)^{2} \boldsymbol{p}\{\boldsymbol{p} \cdot[(\hat{\boldsymbol{p}} \times \boldsymbol{\nabla}) \times \boldsymbol{\nabla}]\} \frac{\partial}{\partial p_{0}}\left(\frac{f_{\mathrm{eq}}}{2 p_{0}^{3}}\right),
\end{aligned}
$$

and $j_{p}^{0}=\hat{\boldsymbol{p}} \cdot \boldsymbol{j}_{p}$, we find that the symmetric part of $\Theta^{0 i}$ contains the vorticity driven thermal Hall effect

$$
q^{i}=\frac{1}{2} \int_{\boldsymbol{p}, \lambda}\left(j_{p}^{0} p^{i}+j_{p}^{i} p^{0}\right)=\frac{\omega^{i j} \partial_{j} \beta}{48 \hbar \beta^{3}}+\mathcal{O}\left(\hbar^{0}\right) .
$$

Combined with Eq. (17), this agrees with Eq. (16). It can also be checked that a similar term in the charge current $\boldsymbol{\tau}=\int_{p, \lambda} \boldsymbol{j}_{p}$ vanishes, in accordance with our expectation in the no-drag frame.

Equivalence between spin hydrodynamics and nondissipative second-order hydrodynamics. - It is well known that the energy-momentum tensor in spin hydrodynamics can be transformed into the symmetric Belinfante-Rosenfeld energy-momentum tensor by a pseudogauge transformation with $\Sigma^{\alpha \mu \nu}=S^{\alpha \mu \nu}$ [16-19],

$$
\begin{aligned}
\tilde{T}^{\mu \nu} & =\Theta^{\mu \nu}+\frac{1}{2} \partial_{\alpha}\left(\Sigma^{\alpha \mu \nu}-\Sigma^{\mu \alpha \nu}-\Sigma^{\nu \alpha \mu}\right) \\
& =\frac{1}{2}\left(\Theta^{\mu \nu}+\Theta^{\nu \mu}\right)-\frac{1}{2} \partial_{\alpha}\left(S^{\mu \alpha \nu}+S^{\nu \alpha \mu}\right) .
\end{aligned}
$$

As a result, the spin tensor no longer appears in the total angular momentum tensor, i.e., $\tilde{S}^{\alpha \mu \nu}=S^{\alpha \mu \nu}-\Sigma^{\alpha \mu \nu}=0$. This leaves the conservation of energy and momentum unchanged, $\partial_{\mu} \tilde{T}^{\mu \nu}=\partial_{\mu} \Theta^{\mu \nu}=0$, and the two descriptions of the system based on each energy-momentum tensor should be equivalent. This suggests that the corresponding hydrodynamic descriptions based on the same premise of local equilibrium, i.e., the spin hydrodynamics and the conventional hydrodynamics, should also be equivalent to each other. We will establish this equivalence and show that the hydrodynamic variables between the two descriptions are related quite nontrivially. In the following, quantities in the spin hydrodynamics will be denoted without the tilde symbol, while those in the conventional hydrodynamics will be written with the tilde symbol.

A central question in showing the equivalence is how the first law of thermodynamics used in hydrodynamics transforms under the pseudogauge transformation. The observation crucial for answering this question is that we can generalize the pseudogauge transformation to the currents of charge and entropy, without affecting their conservation:

$$
\begin{aligned}
& \tilde{j}^{\mu}=j^{\mu}-\partial_{\nu}\left(\frac{a}{2 \chi} S^{\mu \nu}\right)=j^{\mu}-\frac{1}{2} \partial_{\nu}\left(a \omega^{\mu \nu}\right), \\
& \tilde{s}^{\mu}=s^{\mu}-\partial_{\nu}\left(\frac{b}{2 \chi} S^{\mu \nu}\right)=s^{\mu}-\frac{1}{2} \partial_{\nu}\left(b \omega^{\mu \nu}\right),
\end{aligned}
$$

with thermodynamic functions $a(\varepsilon, n), b(\varepsilon, n)$. An intuitive understanding of the physics of these transformations is obtained by noting that the spatial part of $-\partial_{\nu}\left(a \omega^{\mu \nu}\right) / 2$ can be interpreted as the magnetization current $\boldsymbol{\nabla} \times \boldsymbol{M}$ with vorticity induced magnetization $\boldsymbol{M}=-a \omega / 2$, i.e., the Barnett effect.

Since the local charge and entropy densities $(n, s)$ are defined by $n=-u_{\mu} j^{\mu}$ and $s=-u_{\mu} s^{\mu}$, respectively, transformations in Eqs. (21) redefine them: $\tilde{n}=n-\Delta n$, $\tilde{s}=s-\Delta s$, where

$\Delta n=-\frac{1}{2} u_{\mu} \partial_{\nu}\left(a \omega^{\mu \nu}\right)=-a \omega_{\mu} \omega^{\mu}, \quad \Delta s=-b \omega_{\mu} \omega^{\mu}$.

Taking $\tilde{T}^{\mu \nu}$ in Eq. (20) obtained from $\Theta^{\mu \nu}$ in the ideal spin hydrodynamics in the previous section with $S^{\alpha \mu \nu}=u^{\alpha} S^{\mu \nu}=\chi u^{\alpha} \omega^{\mu \nu}$, we work out Landau's condition for the local energy density and the fluid velocity, $\tilde{T}^{\mu \nu} \tilde{u}_{\nu}=-\tilde{\varepsilon} \tilde{u}^{\mu}$, to obtain $\tilde{\varepsilon}$ and $\tilde{u}^{\mu}$ as $\tilde{\varepsilon}=\varepsilon-\Delta \varepsilon$, $\tilde{u}^{\mu}=u^{\mu}-\Delta u^{\mu}$ with

$$
\Delta \varepsilon=2 \chi \omega_{\mu} \omega^{\mu}, \quad \Delta u^{\mu}=-\frac{1}{2 \beta w} \Delta_{\alpha}^{\mu} \partial_{\lambda}\left(\beta \chi \omega^{\alpha \lambda}\right) .
$$


In addition, we allow a redefinition of pressure $\tilde{p}=p-\Delta p$ with $\Delta p=2 a_{0} \omega_{\mu} \omega^{\mu}$, where $a_{0}$ is a free thermodynamic function. In terms of these variables, the energy-momentum tensor in conventional hydrodynamics reads

$$
\tilde{T}^{\mu \nu}=\tilde{\varepsilon} \tilde{u}^{\mu} \tilde{u}^{\nu}+\tilde{p} \tilde{\Delta}^{\mu \nu}+\tilde{\tau}^{\mu \nu},
$$

where $\tilde{\tau}^{\mu \nu}$ denotes certain second-order transport terms

$\tilde{\tau}^{\mu \nu}=\frac{1}{2} \chi\left[\left(\theta_{\alpha}^{\mu}+\omega_{\alpha}^{\mu}\right) \omega^{\alpha \nu}+(\mu \leftrightarrow \nu)\right]+2 a_{0} \Delta^{\mu \nu} \omega_{\lambda} \omega^{\lambda}$.

Similarly, the charge and the entropy currents in conventional hydrodynamics are given by

$$
\begin{gathered}
\tilde{j}^{\mu}=\tilde{n} \tilde{u}^{\mu}-\frac{n}{2 \beta w} \Delta_{\lambda}^{\mu} \partial_{\nu}\left(\beta \chi \omega^{\lambda \nu}\right)-\frac{1}{2} \Delta_{\lambda}^{\mu} \partial_{\nu}\left(a \omega^{\lambda \nu}\right), \\
\tilde{s}^{\mu}=\tilde{s} \tilde{u}^{\mu}-\frac{s \Delta_{\lambda}^{\mu} \partial_{\nu}\left(\beta \chi \omega^{\lambda \nu}\right)}{2 \beta w}+\frac{n \chi \omega^{\mu \nu} \partial_{\nu} \alpha}{2 w}-\frac{\Delta_{\lambda}^{\mu} \partial_{\nu}\left(b \omega^{\lambda \nu}\right)}{2},
\end{gathered}
$$

with other second-order transport terms. A similar observation was made in Ref. [33]. It should be emphasized that the ideal limit of spin hydrodynamics with $\partial_{\mu} s^{\mu}=0$ that we start with guarantees that the conventional hydrodynamics with the above second-order transport terms is also ideal, i.e., $\partial_{\mu} \tilde{s}^{\mu}=0$.

However, to make conventional hydrodynamics truly conventional, the thermodynamics relation of spin hydrodynamics in Eq. (14) should transform into conventional thermodynamic relations

$$
d \tilde{s}=\tilde{\beta} d \tilde{\varepsilon}-\tilde{\alpha} d \tilde{n}, \quad \tilde{s}=\tilde{\beta}(\tilde{\varepsilon}+\tilde{p})-\tilde{\alpha} \tilde{n} .
$$

We now show that there exists a unique choice of $(a, b)$ to achieve this equivalence, with $(a, b)$ expressed in terms of $\left(\chi, a_{0}\right)$ without any integrations.

We start from the entropy density $s$ in the spin hydrodynamics as a function of density variables $s(\varepsilon, n, \sigma)$ where $S^{\mu} \equiv \epsilon^{\mu \nu \alpha \beta} u_{\nu} S_{\alpha \beta} / 2$ and $\sigma \equiv S_{\mu} S^{\mu} / 2$. The first law of thermodynamics in Eq. (14), $d s=\beta d \varepsilon-\alpha d n-\beta \gamma_{\mu \nu} d S^{\mu \nu} / 2=$ $\beta d \varepsilon-\alpha d n-\beta \gamma_{\mu} d S^{\mu}$, then gives us $\beta \equiv(\partial s / \partial \varepsilon)_{n, \sigma}, \quad \alpha \equiv$ $-(\partial s / \partial n)_{\varepsilon, \sigma}$ and $\beta \gamma_{\mu} \equiv-(\partial s / \partial \sigma)_{\varepsilon, n} S^{\mu}$. In local equilibrium, $\gamma_{\mu}=\omega_{\mu}$, and the spin susceptibility is identified as $\chi \equiv-\beta(\partial s / \partial \sigma)_{\varepsilon, n}^{-1}$ from $S^{\mu}=\chi \omega^{\mu}$.

To find the first law of thermodynamics in conventional hydrodynamics, we express $\tilde{s}$ in terms of the variables in conventional hydrodynamics as

$$
\tilde{s}\left(\tilde{\varepsilon}, \tilde{n}, \omega_{\mu} \omega^{\mu}\right)=s(\tilde{\varepsilon}+\Delta \varepsilon, \tilde{n}+\Delta n, \sigma)-\Delta s,
$$

where $\sigma=\chi^{2} \omega_{\mu} \omega^{\mu} / 2$, with the same function $s$ and $(\Delta \varepsilon, \Delta n, \Delta s)$ given by Eqs. (22) and (23).

It is now straightforward to find the first law of thermodynamics:

$$
d \tilde{s}=\tilde{\beta} d \tilde{\varepsilon}-\tilde{\alpha} d \tilde{n}+A \omega_{\mu} d \omega^{\mu}
$$

with

$$
\begin{gathered}
\tilde{\beta}=\beta+\left(\beta \chi_{\varepsilon}+b_{\varepsilon}+\alpha a_{\varepsilon}\right) \omega_{\mu} \omega^{\mu}, \\
\tilde{\alpha}=\alpha-\left(\beta \chi_{n}+b_{n}+\alpha a_{n}\right) \omega_{\mu} \omega^{\mu}, \\
A=3 \beta \chi+2 \alpha a+2 b,
\end{gathered}
$$

where $f_{n} \equiv(\partial f / \partial n)_{\varepsilon}$ and $f_{\varepsilon} \equiv(\partial f / \partial \varepsilon)_{n}$. From $s=$ $\beta(\varepsilon+p)-\alpha n-\beta \chi \omega_{\mu} \omega^{\mu}$ in (14), we also find straightforwardly

$$
\tilde{s}=\tilde{\beta}(\tilde{\varepsilon}+\tilde{p})-\tilde{\alpha} \tilde{n}+B \omega_{\mu} \omega^{\mu},
$$

with

$$
\begin{aligned}
B= & \beta \chi+\alpha a+b-w\left(\beta \chi_{\varepsilon}+b_{\varepsilon}+\alpha a_{\varepsilon}\right) \\
& -n\left(\beta \chi_{n}+b_{n}+\alpha a_{n}\right)+2 \beta a_{0} .
\end{aligned}
$$

The conventional thermodynamics relations in Eq. (28) are obtained by imposing the conditions $A=B=0$. It is easy to see that these conditions determine $(a, b)$ in terms of $\left(\chi, a_{0}\right)$ without any integrations, and we skip their explicit expressions.

With $(a, b)$ given in terms of $\left(\chi, a_{0}\right)$, we see that all second-order transport coefficients in the energy-momentum tensor, Eq. (25); in the charge current, Eq. (26); and in the entropy current, Eq. (27) can be expressed in terms of two free thermodynamic functions $\left(\chi, a_{0}\right)$. With the identification of $a_{1}=\chi$, one can nontrivially check that these second-order transport coefficients agree precisely with those we find in the nondissipative second-order hydrodynamics in the previous section once they are also expressed in terms of $\left(a_{1}, a_{0}\right)$. The conditions $A=0$ and $B=0$ correspond to the constraint $C^{(5)}=0$ and a linear combination of $C^{(i)}=0$, respectively. In the special case of a conformal system, condition $B=0$ follows from $A=0$ and conformality. This completes the proof that the ideal spin hydrodynamics is equivalent to the nondissipative second-order hydrodynamics by pseudogauge transformation.

Conclusion and discussion.-In this Letter, we introduce a novel power counting scheme for gradients of hydrodynamic variables and discover nontrivial constraints on certain nondissipative second-order transport coefficients imposed by the second law of thermodynamics. We also show that the spin hydrodynamics and the conventional hydrodynamics with these second-order transport coefficients are two equivalent descriptions of the same system related by pseudogauge transformation. In a more concrete form, one can express the hydrodynamic variables in one description in terms of those in the other description.

Furthermore, one can construct infinitely many equivalent spin hydrodynamics descriptions for the same system by performing pseudogauge transformations using an arbitrary fraction of the spin tensor, i.e., with $\Sigma^{\alpha \mu \nu}=t S^{\alpha \mu \nu}$, 
where $t \neq 1$. This transformation changes the spin susceptibility $\chi \rightarrow(1-t) \chi \equiv \chi(t)$ in thermodynamic relations, while $a_{1}(t)+\chi(t)$ remains invariant. The other secondorder transport coefficients are related to $a_{1}(t)$ by Eq. (10). The conventional hydrodynamics is a special choice in this infinite family corresponding to $t=1$. In general, the vorticity driven thermal Hall effect is given by Eq. (16) with $\chi \rightarrow a_{1}(t)+\chi(t)$.

What is the meaning of spin densities in different but equivalent spin hydrodynamics descriptions? Our results naturally suggest that the answer to this question cannot be found within hydrodynamics itself. For example, different species of microscopic constituents could carry their own spins, and it is a matter of choice what to include in the hydrodynamic description. Different choices describe the same system, while the nondissipative second-order transport coefficients corresponding to each choice are related in the specific way we described.

Finally, it would be interesting and important for practical applications to study stability and causality issues in the class of ideal hydrodynamic theories we considered, which would necessitate including dissipative terms.

We thank Masaru Hongo, Xu-Guang Huang, and Enrico Speranza for discussions and P. Kovtun for bringing Refs. [20-23] to our attention. This Letter is supported by the U.S. Department of Energy, Office of Science, Office of Nuclear Physics, Grant No. DE-FG0201ER41195, and within the framework of the Beam Energy Scan Theory (BEST) Topical Collaboration.

*sli72@uic.edu

†misha@uic.edu

thyee@uic.edu

[1] L. Landau and E. Lifshitz, Fluid Mechanics, Vol. 6 of Course of Theoretical Physics (Elsevier Science, New York, 2013).

[2] S. Jeon and U. Heinz, Introduction to hydrodynamics, Int. J. Mod. Phys. E 24, 1530010 (2015).

[3] P. Romatschke and U. Romatschke, Relativistic fluid dynamics in and out of equilibrium - ten years of progress in theory and numerical simulations of nuclear collisions, arXiv:1712.05815.

[4] W. Florkowski, B. Friman, A. Jaiswal, and E. Speranza, Relativistic fluid dynamics with spin, Phys. Rev. C 97, 041901(R) (2018).

[5] K. Hattori, M. Hongo, X.-G. Huang, M. Matsuo, and H. Taya, Fate of spin polarization in a relativistic fluid: An entropy-current analysis, Phys. Lett. B 795, 100 (2019).

[6] A. Gallegos and U. Gürsoy, Holographic spin liquids and Lovelock Chern-Simons gravity, J. High Energy Phys. 11 (2020) 151.

[7] L. Adamczyk et al. (STAR Collaboration), Global $\Lambda$ hyperon polarization in nuclear collisions: Evidence for the most vortical fluid, Nature (London) 548, 62 (2017).
[8] Z.-T. Liang and X.-N. Wang, Globally Polarized QuarkGluon Plasma in Non-Central A + A Collisions, Phys. Rev. Lett. 94, 102301 (2005); Erratum, Phys. Rev. Lett. 96, 039901 (2006).

[9] B. Betz, M. Gyulassy, and G. Torrieri, Polarization probes of vorticity in heavy ion collisions, Phys. Rev. C 76, 044901 (2007).

[10] F. Becattini, F. Piccinini, and J. Rizzo, Angular momentum conservation in heavy ion collisions at very high energy, Phys. Rev. C 77, 024906 (2008).

[11] X.-G. Huang, P. Huovinen, and X.-N. Wang, Quark polarization in a viscous quark-gluon plasma, Phys. Rev. C 84, 054910 (2011).

[12] Y. Jiang, Z.-W. Lin, and J. Liao, Rotating quark-gluon plasma in relativistic heavy ion collisions, Phys. Rev. C 94, 044910 (2016); Erratum, Phys. Rev. C 95, 049904 (2017).

[13] Y. Sun and C. M. Ko, $\Lambda$ hyperon polarization in relativistic heavy ion collisions from a chiral kinetic approach, Phys. Rev. C 96, 024906 (2017).

[14] Certain variants of spin hydrodynamics [5] could describe off-equilibrium dynamics of spin polarization in a system where the relaxation time of spin polarization is much slower than other microscopic timescales. Similar extensions of hydrodynamics by nonhydrodynamic, but nevertheless parametrically slow, variables have been termed Hydro+ [15].

[15] M. Stephanov and Y. Yin, Hydrodynamics with parametric slowing down and fluctuations near the critical point, Phys. Rev. D 98, 036006 (2018).

[16] F. Becattini and L. Tinti, Nonequilibrium thermodynamical inequivalence of quantum stress-energy and spin tensors, Phys. Rev. D 87, 025029 (2013).

[17] F. Becattini, W. Florkowski, and E. Speranza, Spin tensor and its role in non-equilibrium thermodynamics, Phys. Lett. B 789, 419 (2019).

[18] W. Florkowski, A. Kumar, and R. Ryblewski, Relativistic hydrodynamics for spin-polarized fluids, Prog. Part. Nucl. Phys. 108, 103709 (2019).

[19] E. Speranza and N. Weickgenannt, Spin tensor and pseudogauges: from nuclear collisions to gravitational physics, Eur. Phys. J. A 57, 155 (2021).

[20] S. Bhattacharyya, Constraints on the second order transport coefficients of an uncharged fluid, J. High Energy Phys. 07 (2012) 104.

[21] K. Jensen, M. Kaminski, P. Kovtun, R. Meyer, A. Ritz, and A. Yarom, Towards Hydrodynamics Without an Entropy Current, Phys. Rev. Lett. 109, 101601 (2012).

[22] N. Banerjee, J. Bhattacharya, S. Bhattacharyya, S. Jain, S. Minwalla, and T. Sharma, Constraints on fluid dynamics from equilibrium partition functions, J. High Energy Phys. 09 (2012) 046.

[23] S. Bhattacharyya, Entropy current from partition function: One example, J. High Energy Phys. 07 (2014) 139.

[24] J. Erdmenger, M. Haack, M. Kaminski, and A. Yarom, Fluid dynamics of R-charged black holes, J. High Energy Phys. 01 (2009) 055.

[25] R. Baier, P. Romatschke, D. T. Son, A. O. Starinets, and M. A. Stephanov, Relativistic viscous hydrodynamics, conformal invariance, and holography, J. High Energy Phys. 04 (2008) 100. 
[26] F. Becattini and L. Tinti, The Ideal relativistic rotating gas as a perfect fluid with spin, Ann. Phys. (Amsterdam) 325, 1566 (2010).

[27] A. Aristova, D. Frenklakh, A. Gorsky, and D. Kharzeev, Vortical susceptibility of finite-density QCD matter, J. High Energy Phys. 10 (2016) 029.

[28] K. Rajagopal and A. V. Sadofyev, Chiral drag force, J. High Energy Phys. 10 (2015) 018.

[29] M. A. Stephanov and H.-U. Yee, No-Drag Frame for Anomalous Chiral Fluid, Phys. Rev. Lett. 116, 122302 (2016).
[30] J.-Y. Chen, D. T. Son, and M. A. Stephanov, Collisions in Chiral Kinetic Theory, Phys. Rev. Lett. 115, 021601 (2015).

[31] J.-Y. Chen, D. T. Son, M. A. Stephanov, H.-U. Yee, and Y. Yin, Lorentz Invariance in Chiral Kinetic Theory, Phys. Rev. Lett. 113, 182302 (2014).

[32] J.-H. Gao, Z.-T. Liang, Q. Wang, and X.-N. Wang, Disentangling covariant Wigner functions for chiral fermions, Phys. Rev. D 98, 036019 (2018).

[33] K. Fukushima and S. Pu, Spin hydrodynamics and symmetric energy-momentum tensors-A current induced by the spin vorticity-, Phys. Lett. B 817, 136346 (2021). 